\title{
Resultados visuales en pacientes con macroprolactinoma tratados con agonistas de dopamina
}

\author{
JOSÉ LUIS CUEVAS ${ }^{1,2}$, VERÓNICA FERNÁNDEZ ${ }^{1,2}$, DAVID ROJAS ${ }^{1,2}$, \\ NELSON WOHLLK ${ }^{1,3}$, ÓSCAR GONZÁLEZ ${ }^{1}$, ESTEBAN TORCHE $^{1}$
}

\section{Visual outcome in patients with macroprolactinoma treated with dopamine agonists}

Background: Dopamine agonists (DA) effectively reduce tumor size of macroprolactinomas, with the consequent improvement of eventual visual impairment. Aim: To study the visual outcomes in patients with macroprolactinoma treated with DA. Material and Methods: Retrospective cohort study which included patients with macroprolactinoma controlled at a Neuroendocrinology and Neuroophthalmology Department between 1997 and 2011, and treated exclusively with DA (bromocriptine or cabergoline). Patients who were operated or had previous radiotherapy and those with an incomplete follow up, were excluded. We analyzed and compared the visual status before and after the beginning of DA treatment. Results: Thirty one patients aged 8 to 59 years, were included. Eighteen patients (58\%) had visual impairment at the moment of diagnosis (group 1) and 13 had no alterations (group 2). Mean follow up was 36.5 months. Fifteen patients from group 1 (83\%) had visual improvement, two remained stable (11\%) and one had a visual deterioration (6\%). In group 2, only one non-compliant patient had a visual deterioration. Conclusions: DAs are effective in the management of neuro-ophthalmic complications associated to macroprolactinomas and should be considered as first choice therapy in these tumors.

(Rev Med Chile 2013; 141: 687-694).

Key words: Dopamine agonist; Prolactinoma; Visual acuity; Visual fields.

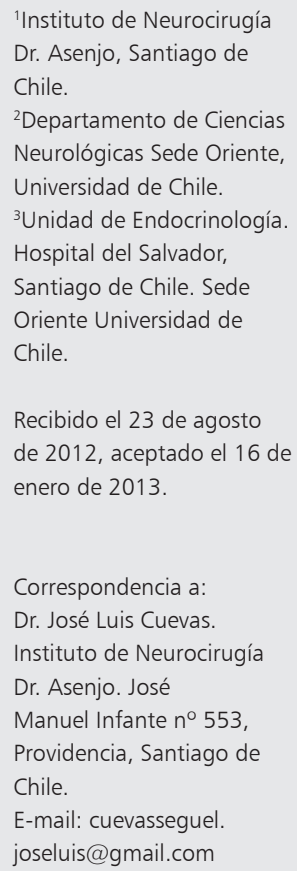

L os prolactinomas son el tipo más común de tumores hipofisarios, dando cuenta de 40\% de todos los adenomas pituitarios ${ }^{1}$. Son más frecuentes en mujeres, con un peak de incidencia entre los 20 y 50 años, edad a la cual la razón es de 10:1. La incidencia se estima en 6 a 10 casos por millón de habitantes por año y la prevalencia en 60-100 casos por millón ${ }^{2-4}$. Esta entidad constituye la principal patología neuroendocrina en la práctica neuroquirúrgica y es también motivo frecuente de consulta a médicos internistas, gínecoobstetras, oftalmólogos, urólogos y neurólogos, dado su amplio espectro de presentación.

Las manifestaciones clínicas de los prolacti- nomas dependen de los efectos neuroendocrinos de la hiperprolactinemia, así como del tamaño tumoral. En $90 \%$ de los casos se trata de tumores pequeños e intraselares o "microadenomas" $(\leq 10 \mathrm{~mm})$ que se manifiestan habitualmente por galactorrea, amenorrea e infertilidad en el caso de mujeres o disminución de la libido e impotencia en hombres, la mayoría signos de hipogonadismo. El 5-10\% pueden crecer y alcanzar un tamaño mayor a $10 \mathrm{~mm}$ denominándose "macroadenomas o macroprolactinomas", en cuyo caso el efecto de masa del tumor puede manifestarse por cefalea, hipopituitarismo (por compresión hipofisiaria o del tallo) o producir deterioro visual secundario 
a compresión quiasmática. Las manifestaciones visuales son variadas e incluyen disminución de la agudeza visual, alteraciones del campo visual (escotomas, cuandrantopsias o clásicamente hemianopsia bitemporal de predominio superior) y alteraciones de la oculomotilidad o ceguera súbita (en contexto de tumores con invasión al seno cavernoso o apoplejía, respectivamente). El diagnóstico se confirma por niveles elevados de prolactina (PRL) en sangre (PRL neta y diluida $>150-200 \mathrm{ng} / \mathrm{mL}$ ) y resonancia magnética $(\mathrm{RM})$ o tomografía computada (TC) de silla turca (con y sin uso de contraste), siendo la RM el examen de elección ${ }^{1,5,6}$. La dilución 1:100 debe hacerse en cualquier tumor hipofisario $>20-30 \mathrm{~mm}$ o macroadenoma de tamaño considerable con prolactina normal o levemente elevada, para evitar el "efecto gancho" (hook effect) ${ }^{1}$.

Los objetivos de la terapia son la normalización de los niveles de PRL, restauración de la función gonadal y reducción el tamaño o efecto de masa tumoral ${ }^{7}$. Los agonistas de dopamina (AD) -bromocriptina y cabergolina- son actualmente la primera línea de tratamiento en prolactinomas y han desplazado a la cirugía como manejo primario de esta condición en las últimas dos décadas. Varios estudios han demostrado la efectividad de los AD en reducir el exceso de PRL y disminuir el tamaño tumoral, con la consiguiente mejoría neuroendocrina y visual, respectivamente ${ }^{1,5,7-11}$. Sin embargo, y pese a existir numerosa literatura respecto a la utilidad de estos fármacos, muchos neurocirujanos aún continúan teniendo una conducta quirúrgica ante pacientes con macroprolactinomas y compromiso visual, dada la creencia popular de que la cirugía es una solución más rápida, segura y efectiva en términos de recuperación visual.

El presente trabajo tiene por objetivo describir los resultados visuales en pacientes con diagnóstico de "macroprolactinoma", que fueron tratados exclusivamente con AD en el Instituto de Neurocirugía Dr. Asenjo (INCA) en los últimos 15 años.

\section{Material y Método}

De forma retrospectiva, se revisó la condición visual de los pacientes con diagnóstico de "macroprolactinoma hipofisiario" controlada en el Instituto de Neurocirugía Dr. Asenjo entre los años 1997 y 2011, y que fueron tratados exclusi- vamente con agonistas de dopamina (cabergolina, bromocriptina, o ambos).

Se incluyeron en el estudio aquellos pacientes que cumplieron con los siguientes criterios: 1) cuadro clínico atribuible a hiperprolactinemia (con o sin deterioro visual al momento del diagnóstico); 2) macroadenoma selar confirmado por RM o TC de silla turca y 3 ) nivel de PRL neta y diluida [1:100] $>150 \mathrm{ng} / \mathrm{mL}$, no atribuible a otras causas. Se excluyeron aquellos pacientes con tratamiento quirúrgico previo, radioterapia, seguimiento incompleto o déficit visual severo atribuible a otras causas oftalmológicas. Todos los pacientes fueron evaluados de forma estandarizada en la Unidad de Neuro-Oftalmología del INCA al momento de su diagnóstico y tuvieron seguimiento neurooftalmológico (NOF) y neuroendocrino luego de iniciado el tratamiento por períodos de tiempo variable. El protocolo de seguimiento NOF consideró evaluación al mes de iniciado el tratamiento médico (cuando había deterioro visual), a los 3 meses, luego cada 6 meses por 1 ó 2 años y anual de por vida. En cada evaluación NOF se valoró estado de la agudeza visual (AV) según tabla de Snellen, campo visual (CV) por campímetro de Goldmann, aspecto de la papila al fondo de ojo y estado de la oculomotilidad. Se consideró como adecuado para los propósitos del estudio contar con al menos una evaluación NOF anterior y otra posterior inicio del tratamiento.

Los pacientes fueron divididos en 2 grupos: 1) pacientes con compromiso visual al momento del diagnóstico (tradicionalmente considerados candidatos a tratamiento quirúrgico) y 2) pacientes sin compromiso visual al momento del diagnóstico (grupo habitualmente tratado con medicamentos pero con riesgo potencial de deterioro visual). Se analizaron y compararon los resultados visuales pre y post tratamiento, de ambos grupos en relación a las variables mencionadas previamente.

\section{Resultados}

En el período de estudio se evaluó a un total de 47 pacientes con diagnóstico de macroprolactinoma. Se analizaron 31 pacientes (16 mujeres y 15 hombres) que cumplían criterios de inclusión, cuya edad promedio fue de 32 años (8-59). Dieciocho pacientes (58\%) tenían compromiso visual al momento del diagnóstico y 13 estaban 
sin deterioro (42\%). Todos los pacientes fueron tratados con bromocriptina, cabergolina, o ambos en dosis variables según prescripción endocrinológica. Veinticinco pacientes $(80,6 \%)$ recibieron cabergolina como tratamiento único, 3 recibieron bromocriptina requiriendo posteriormente cabergolina por falta de respuesta $(9,7 \%)$ y 3 fueron tratados con bromocriptina durante todo el seguimiento $(9,7 \%)$. El seguimiento neurooftalmológico promedio fue de 36,5 meses.

Entre los pacientes con compromiso visual al momento del diagnóstico (Grupo 1, $\mathrm{n}=18$ ), 12 tenían disminución de la $\mathrm{AV}$ asociado a compromiso de CV (1 tenía paresia de III NC izquierdo) y 6 tenían sólo compromiso de CV. Quince de los 18 pacientes $(83 \%)$ presentaron mejoría visual en grados variables durante el seguimiento, 2 se mantuvieron estables con su déficit (11\%) y 1 tuvo deterioro visual (6\%), pese a terapia bien llevada (caso ${ }^{\circ} 12$ ).

Siete pacientes $(38,8 \%)$ lograron visión completamente normal (AV y CV) a 9,7 meses de seguimiento en promedio, y la mejoría visual pudo apreciarse al mes de iniciado el tratamiento en un caso y 20 meses después en otros dos casos. En 8 pacientes del grupo $1(44,4 \%)$ la mejoría visual fue parcial (5 pacientes tuvieron mejoría de AV pero persistieron con déficit de CV de menor grado y 3 pacientes mejoraron su $\mathrm{CV}$ pero persistieron con déficit de AV).

De los pacientes con compromiso de AV $(\mathrm{n}=12), 50 \%$ tuvo mejoría completa. La presencia de atrofia papilar en los casos no 7 y 10 se asoció a recuperación visual de menor cuantía. La única paciente que presentó deterioro visual a pesar del tratamiento con $\mathrm{AD}$ (caso $\mathrm{n}^{\circ} 12$ ) tenía historia de disminución visual de 6 meses. Posterior al inicio de la terapia, se objetivó disminución del tamaño tumoral en la RM de silla turca, pese a lo cual se instaló déficit visual y signos de atrofia papilar. Los cambios neuro-oftalmológicos se resumen en las Figuras 1 y 2. Las Figuras 3 y 4 ejemplifican la disminución del tamaño tumoral y la mejoría del $\mathrm{CV}$, respectivamente.
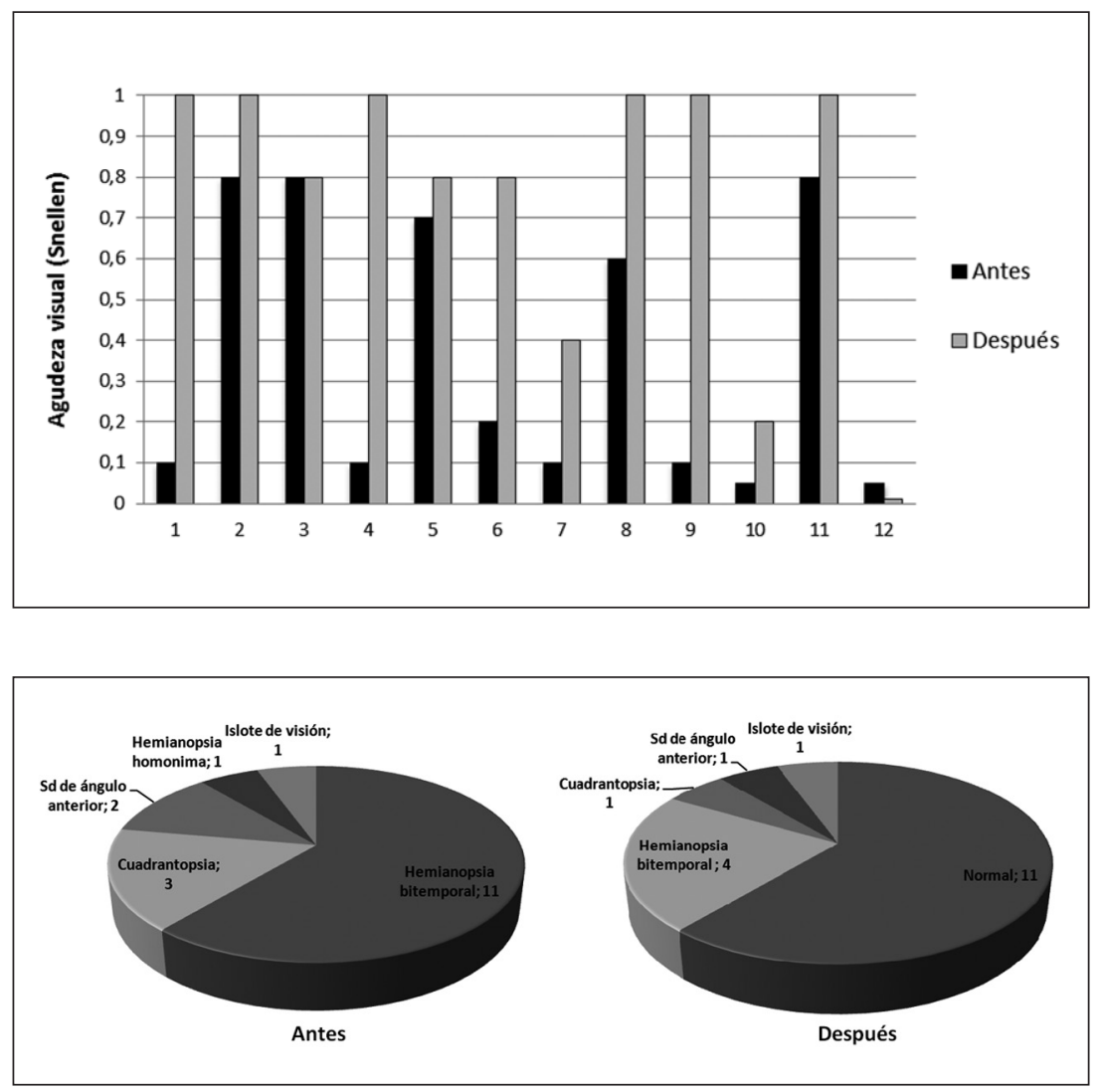

Figura 1. Agudeza visual pre y post tratamiento con $A D$ en pacientes con compromiso de AV al momento del diagnóstico (Grupo 1, $\left.\mathrm{n}^{\circ}=12\right)^{*}$. *Los cambios en la $\mathrm{AV}$ representados corresponden al ojo más comprometido.
Figura 2. Distribución de los defectos de campo visual antes y después del tratamiento con AD (Grupo 1, n 18). 
En el grupo de pacientes que no tenían compromiso visual al momento del diagnóstico (Grupo 2, $\mathrm{n}^{\circ}=13$ ), 12 se mantuvieron estables y sólo 1 paciente presentó deterioro. Este caso correspondió al de una paciente con controles irregulares y con mala adhesión al tratamiento con cabergolina. Finalmente, tras discontinuar su terapia por 6 años presentó apoplejía hipofisiaria, evidenciándose en ese momento daño de nervio óptico izquierdo.

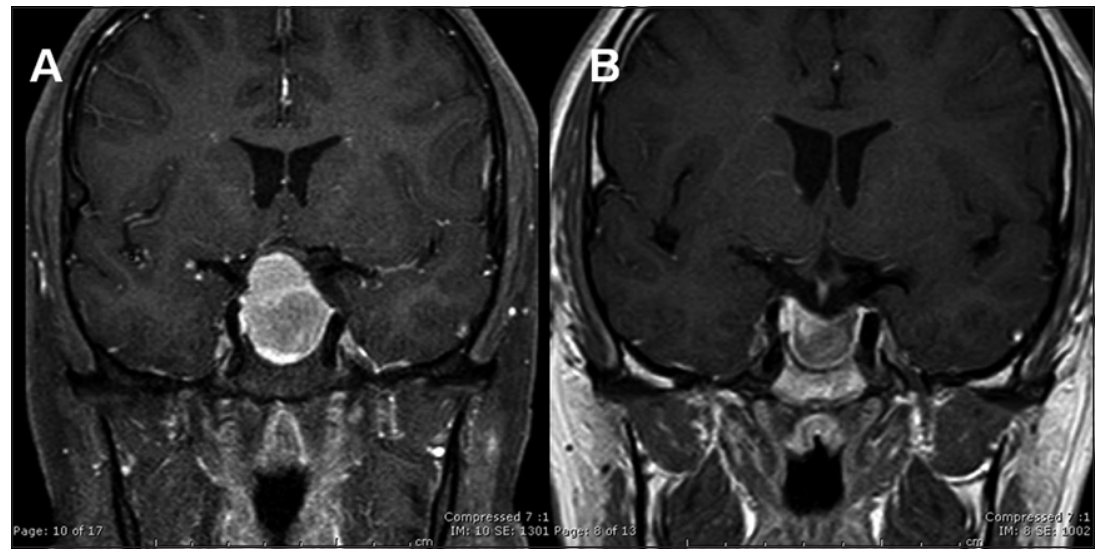

Figura 3. Resonancia Magnética pre y post tratamiento con AD. A. Macroprolactinoma de $33 \mathrm{~mm}$ que comprime quiasma óptico. B. Macroprolactinoma de $11 \mathrm{~mm}$ tras 6 meses de tratamiento con $\mathrm{AD}(\mathrm{B})$.

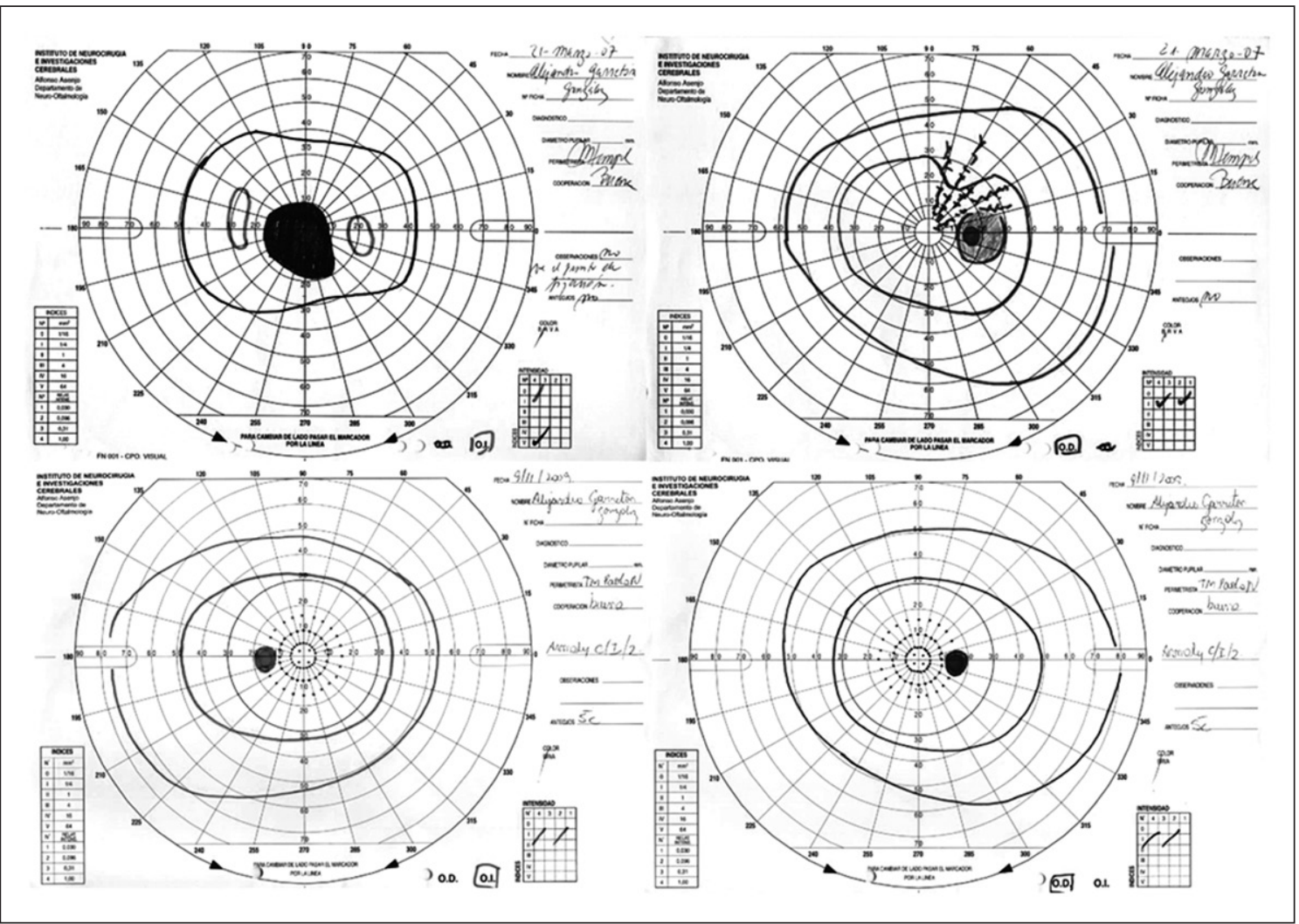

Figura 4. Campo visual pre y post tratamiento con AD. Síndrome de ángulo anterior del quiasma, con mayor afección del ojo izquierdo (CV superiores). Luego de 20 meses con AD el paciente recupera el defecto completamente (CV inferiores). 
En esta serie hubo 1 paciente con apoplejía hipofisiaria y compromiso neuro-oftalmológico al momento del diagnóstico, sin embargo, su manejo inicial se realizó en otro centro. Esta paciente no fue tratada quirúrgicamente por razones que desconocemos y recibió cabergolina. A los 6 meses de seguimiento mejoró su AV y normalizó su CV.

\section{Discusión}

El tratamiento y pronóstico de los prolactinomas ha mejorado sustancialmente en las últimas décadas. La cirugía por vía trans-esfenoidal -considerada por años como el tratamiento de elección en prolactinomas-, si bien permite una resección tumoral precisa, preservando la función glandular con una baja proporción de complicaciones, habitualmente no constituye un tratamiento definitivo a largo plazo, asociándose a un elevado porcentaje de recurrencia de hiperprolactinemia que se sitúa cercano a $20 \%$, con un rango de 12 a 50\% según diferentes publicaciones ${ }^{1,12-17}$. Los porcentajes de cura son aun más pobres en el caso de macroprolactinomas o prolactinomas con extensión hacia el seno cavernoso, los que esencialmente no pueden ser resecados mediante cirugía ${ }^{1}$.

Los AD son hoy en día la terapia de primera línea en la mayoría de los pacientes con prolactinomas ${ }^{1,5,8}$. Los AD más utilizados son la bromocriptina y cabergolina. Ambos son derivados del ergot con acción agonista sobre los receptores D2 ${ }^{5}$. La bromocriptina posee una vida media corta, por lo que debe administrarse 2 ó 3 veces al día y la dosis terapéutica va desde los 2,5 mg hasta los 30 mg diarios (en casos resistentes). A diferencia de la cabergolina, se asocia con mayor frecuencia a efectos adversos (ej. náuseas, vómitos, hipotensión postural, cefalea y vértigo). La cabergolina puede iniciarse en dosis de 0,25-0,5 mg administrados 1 ó 2 veces por semana, con dosis de mantención de hasta $3 \mathrm{mg}$ semanales. Sólo 1-5\% de los pacientes presentan efectos adversos significativos, siendo la fibrosis de las válvulas aórtica, mitral y tricuspídea el más temido. Su mayor inconveniente es el elevado costo, comparado con la bromocriptina ${ }^{1,5,7}$.

La efectividad de los $\mathrm{AD}$ ha sido evaluada en varios estudios. La bromocriptina controla la hiperprolactinemia, restaura la función gonadal y disminuye el tamaño tumoral en $80-90 \%$ de los pacientes con microprolactinomas y $70 \%$ de los macroprolactinomas ${ }^{18}$. La cabergolina ha demostrado ser superior a la bromocriptina, logrando normalizar los niveles de PRL en 92\% de los pacientes con hiperprolactinemia idiopática o microprolactinoma y $77 \%$ de los macroprolactinomas ${ }^{19}$. Estudios comparativos sobre estos fármacos han obtenido resultados similares, confirmando la superioridad de la cabergolina sobre la bromocriptina ${ }^{20,21}$. Basado en estos resultados y la baja frecuencia de efectos adversos, la cabergolina está claramente indicada como tratamiento de elección en macroprolactinomas ${ }^{22}$.

Los $\mathrm{AD}$ han demostrado ser útiles en el manejo de los síntomas visuales en pacientes con macroprolactinoma. La mejoría visual se obtiene en $67 \%-100 \%$ de los casos con resultados objetivables incluso en la primera o segunda semana de tratamiento, ya sea con bromocriptina o cabergolina ${ }^{2,19,23-27}$. Se han obtenido buenos resultados con $\mathrm{AD}$ también en prolactinomas gigantes $(>4 \mathrm{~cm})$ y prolactinomas invasores. Shrivastava et $\mathrm{al}^{12}$ reportaron mejoría visual en $100 \%$ (8/8) de los pacientes con prolactinoma gigante tratados exclusivamente con bromocriptina ( 5 de los cuales lograron visión normal). Similarmente, Acharya ${ }^{28}$, Shimon $^{29}$ y Corsello et a ${ }^{30}$ obtuvieron mejoría visual en $100 \%$, $89 \%$ y $86 \%$ de los pacientes con prolactinoma gigante tratados con cabergolina, respectivamente. Yang et $\mathrm{al}^{31}$ evaluaron 36 pacientes con prolactinoma invasor y compromiso visual, reportando mejoría en $94 \%$ de los pacientes tratados con bromocriptina. Estudios más recientes han alertado respecto a la posibilidad de empeoramiento visual tardío relacionado con herniación quiasmática secundario a $\mathrm{AD}$, lo que podría ocurrir hasta en $10 \%$ de los pacientes, aunque esta complicación puede responder a la suspensión del fármaco ${ }^{32}$.

En nuestro estudio se confirma la buena respuesta al tratamiento farmacológico con $\mathrm{AD}$. De los pacientes con compromiso visual inicial, $83 \%$ logra recuperar visión (50\% lograron mejoría completa de AV conservando defectos campimétricos menores y $38,8 \%$ lograron mejoría completa de $\mathrm{AV}$ y CV), resultados comparables a los estudios previamente publicados. Los cambios neurooftalmológicos pudieron apreciarse tempranamente en la mayoría de los pacientes, aunque en algunos casos la mejoría se presentó tardíamente. En el único caso en que se documentó deterioro visual pese a tratamiento correcto $\left(\right.$ caso $\left.^{\circ} 12\right)$ no se comprobó asociación con herniación quias- 
mática, por lo que el deterioro lo atribuimos a la cronicidad de la pérdida visual antes de iniciar el tratamiento con AD.

La utilidad de la cirugía en el manejo de los síntomas visuales es conocida. La mayoría de las series quirúrgicas publicadas, no obstante, han evaluado resultados visuales en macroadenomas no funcionantes. Estos estudios reportan mejoría visual en hasta $75-95 \%$ de los pacientes sometidos a cirugía trans-esfenoidal o trans-cranial ${ }^{33-38}$. En el caso de macroprolactinomas, la mejoría visual se obtiene en 64 a $69 \%$ de los $\operatorname{casos}^{39,40}$. Estos resultados sugieren que en el manejo de los síntomas visuales en el contexto particular de macroprolactinomas los AD podrían ser superiores al tratamiento quirúrgico.

Los factores pronósticos asociados a recuperación visual han sido menos estudiados. Se ha citado a la duración de los síntomas, severidad de la pérdida visual, presencia o ausencia de atrofia óptica, edad y tamaño del tumor, entre otros ${ }^{6}$. De todos ellos, pareciera que la presencia de atrofia óptica al momento del diagnóstico es uno de los factores más relevantes a la hora de establecer un pronóstico, independiente del tratamiento que se otorgue ${ }^{33,35,36,38}$. En nuestra serie, los peores resultados visuales se presentaron en aquellos pacientes con atrofia óptica, lo que corrobora los hallazgos de estudios previos.

Pese a lo promisorio de estos medicamentos, cerca de $10 \%$ de los microprolactinomas y hasta $18 \%$ de los macroprolactinomas tratados con $\mathrm{AD}$ no responden a tratamiento ${ }^{21}$, en cuyo caso el tratamiento quirúrgico está plenamente justificado. Otras indicaciones quirúrgicas en las cuales existe consenso en la actualidad se detallan en la Tabla 1.

En los últimos años se ha cuestionado la seguridad de los AD (cabergolina y pergolida) dada la asociación existente entre estos fármacos y enfermedad valvular cardiaca, basado principalmente en estudios observacionales de pacientes con enfermedad de Parkinson tratados con altas dosis acumulativas de $\mathrm{AD}^{5,7}$. Sin embargo, un meta-análisis reciente revisó nueve estudios con un total 639 pacientes con prolactinoma tratados con bajas dosis de cabergolina, y no encontró asociación significativa entre cabergolina en dosis bajas y valvulopatía ${ }^{41}$. Estos datos proporcionan razonable evidencia para la tranquilidad de los clínicos que tratan pacientes con prolactinomas, aunque mientras no existan ensayos prospectivos es aconsejable una adecuada evaluación cardiológica ante signos sugerentes de enfermedad valvular ${ }^{5}$. En nuestro estudio, no se registraron complicaciones cardiacas atribuibles al uso de AD.

\section{Conclusiones}

Los $\mathrm{AD}$ son efectivos en el tratamiento de los síntomas visuales en macroprolactinomas. Nuestros resultados, así como los de otros estudios, demuestran excelente porcentaje de respuesta clínica, lo que es objetivable incluso a pocos días de iniciado el tratamiento. Si además se considera que estos fármacos han probado mayor utilidad en el tratamiento de la hiperprolactinemia e hipogonadismo asociado a prolactinomas, con baja proporción de recurrencia comparado con la cirugía, los $\mathrm{AD}$ deben considerarse la terapia de primera elección en pacientes con prolactinomas. El rol de la cirugía sigue plenamente vigente en casos de refractariedad a tratamiento médico, deterioro visual agudo en contexto de apoplejía hipofisiaria u otros escenarios clínicos en los que existe consenso de la mayor utilidad del tratamiento quirúrgico por sobre el manejo farmacológico. La evaluación multidisciplinaria entre el endocrinólogo, neuro-oftalmólogo y neurocirujano es

\section{Tabla 1. Indicaciones de cirugía en prolactinomas ${ }^{1,8,22}$}

Prolactinoma resistente a agonistas de dopamina

- Crecimiento del tumor pese a tratamiento médico óptimo

- Inadecuada reducción de PRL para restaurar función gonadal pese a tratamiento médico óptimo

Intolerancia a los agonistas de dopamina

Apoplejía con síntomas neurológicos en macroprolactinomas

Compresión quiasmática persistente pese a tratamiento óptimo con agonistas de dopamina

Fístula de LCR (rinorraquia) por erosión del tumor en el seno esfenoidal

Macroprolactinomas quísticos (generalmente no responden a $A D$ )

Contraindicación para el uso de agonistas de dopamina (ej. ciertas patologías psiquiátricas)

Preferencia personal de tratamiento quirúrgico 
la piedra angular para lograr buenos resultados clínicos en estos pacientes.

\section{Referencias}

1. Casanueva F, Molitch M, Schlechte J, Abs R, Bonert V, Bronstein M, et al. Guidelines of the Pituitary Society for the diagnosis and management of prolactinomas. Clinical Endocrinology 2006; 65: 265-73.

2. Colao A, Sarno AD, Cappabianca P, Briganti F, Pivonello $\mathrm{R}$, Somma CD, et al. Gender differences in the prevalence, clinical features and response to cabergoline in hyperprolactinemia. Eur J Endocrinol 2003; 148: 325-31.

3. Mindermann T, Wilson CB. Age-related and gender-related occurrence of pituitary adenomas. Clin Endocrinol (Oxf) 1994; 41: 359-64.

4. Ciccarelli A, Daly A, Beckers A. The epidemiology of prolactinomas. Pituitary 2005; 8: 3-6.

5. Ogilvie C, Milsom S. Dopamine agonists in the treatment of prolactinomas: are they still the first choice? Intern Med J 2010; 41 (2): 156-61.

6. Gittinger J. Tumors of the pituitary gland. En: Miller N, Newman N, editors. Walsh \& Hoyts. Clinical NeuroOphthalmology. Baltimore, Meriland. Lippincott Williams \& Wilkins, 6º edition, 2005. p. 1531-46.

7. Colao A, Savastano S. Medical treatment of prolactinomas. Nat Rev Endocrinol 2011; 7: 267-78.

8. Klibanski A. Prolactinomas. N Engl J Med 2010; 362: 1219-26.

9. Molitch M, Elton R, Blackwell R, Caldwell B, Chang $\mathrm{R}$, Jaffe $\mathrm{R}$, et al. Bromocriptine as primary therapy for prolactin-secreting macroadenomas: results of a prospective multicenter study. J Clin Endocrinol Metab 1985; 60: 698-705.

10. Bevan J, Webster J, Burke C, Scanlon M. Dopamine agonists and pituitary tumour shrinkage. Endocrine Reviews 1992; 13: 220-40.

11. Acharya S, Gopal R, Menon P, Bandgar T, Shah N. Giant prolactinomas and effectiveness of medical management. Endocr Pract 2010; 16 (1): 42-6.

12. Shrivastava R, Argiteanu M, King W, Post K. Giant prolactinomas: clinical management and long-term follow up. J Neurosurg 2002; 97: 299-306.

13. Fahlbusch R, Buchfelder M. Present status of neurosurgery in the treatment of prolactinomas. Neurosurg Rev 1985; 8: 195-205.

14. Tyrrell JB, Lamborn KR, Hannegan LT, Applebury CB, Wilson CB. Transsphenoidal microsurgical therapy of prolactinomas: initial outcomes and long-term results. Neurosurgery 1999; 44: 254-63.

15. Serri O, Rasio E, Beauregard H, Hardy J, Somma M. Re- currence of hiperprolactinemia after selective transsphenoidal adenomectomy in women with prolactinoma. $\mathrm{N}$ Engl J Med 1983; 309: 280-3.

16. Losa M, Mortini P, Barzaghi R, Gioia L, Giovanelli M. Surgical treatment of prolactin-secreting pituitary adenomas: early results and long-term outcome. J Clin Endocrinol Metab 2002; 87 (7): 3180-6.

17. Kreutzer J, Buslei R, Wallaschofski H, Hofmann B, Nimsky C, Fahlbusch R, et al. Operative treatment of prolactinomas: indications and results in a current consecutive series of 212 patients. Eur J Endocrinol 2008; 158: 11-8.

18. Colao A, di Sarno A, Pivonello R, di Somma C, Lombardi G. Dopamine receptor agonists for treating prolactinomas. Expert Opin Investig Drugs 2002; 11: 787-800.

19. Verhelst J, Abs R, Maiter D, Van den Bruel A, Vandeweghe $\mathrm{M}$, Velkeniers B, et al. Cabergoline in the treatment of hyperprolactinemia: a study in 455 patients. J Clin Endocrinol Metab 1999; 84: 2518-22.

20. Webster J, Piscitelli G, Polli A, Ferrari CI, Ismail I, Scanlon MF. A comparison of cabergoline and bromocriptine in the treatment of hyperprolactinemic amenorrhea. $\mathrm{N}$ Engl J Med 1994; 331: 904-9.

21. Di Sarno A, Landi ML, Cappabianca P, Di Salle F, Rossi FW, Pivonello R, et al. Resistance to cabergoline as compared with bromocriptine in hyperprolactinemia: prevalence, clinical definition, and therapeutic strategy. J Clin Endocrinol Metab 2001; 86: 5256-61.

22. Gillam M, Molitch M, Lombardi G, Colao A. Advances in the treatment of prolactinomas. Endocr Rev 2006; 27: 485-534.

23. Moster M, Savino P, Schatz N, Snyder P, Sergott R, Bosley T. Visual function in prolactinoma patients treated with bromocriptine. Ophthalmology 1985; 92 (10): 1332-41.

24. Mbanya J, Mendelow A, Crawford P, Hall K, Dewar J, Kendall-Taylor P. Rapid resolution of visual abnormalities with medical therapy alone inpatients with large prolactinomas. Br J Neurosurg 1993; 7 (5): 519-27.

25. Ferrari CI, Abs R, Bevan JS, Brabant G, Ciccarelli E, Motta T, et al. Treatment of macroprolactinoma with cabergoline: a study of 85 patients. Clin Endocrinol (Oxf) 1997; 46 (4): 409-13.

26. Pontikides N, Krassas GE, Nikopoulou E. Cabergoline as a first-line treatment in newly diagnosed macroprolactinomas. Pituitary 2000; 2 (4): 277-81.

27. Colao A, Vitale G, Cappabianca P, Briganti F, Ciccarelli A, De Rosa M, et al. Outcome of cabergoline treatment in men with prolactinoma: effects of a 24-month treatment on prolactin levels, tumor mass, recovery of pituitary function, and semen analysis. J Clin Endocrinol Metab 2004; 89 (4): 1704-11.

28. Acharya S, Gopal R, Menon P, Bandgar T, Shah N. Giant 
prolactinoma and effectiveness of medical management. Endocr Pract 2010; 16 (1): 42-6.

29. Shimon I, Benbassat C, Hadani M. Effectiveness of long-term cabergolina treatment for giant prolactinoma: study of 12 men. Eur J Endocrinol 2007; 156 (2): 225-31.

30. Corsello SM, Ubertini G, Altomare M, Lovicu RM, Migneco MG, Rota CA, et al. Giant prolactinomas in men: efficacy of cabergoline treatment. Clin Endocrinol (Oxf) 2003; 58 (5): 662-70.

31. Yang MS, Hong JW, Lee SK, Lee EJ, Kim SH. Clinical management and outcome of 36 invasive prolactinomas treated with dopamine agonist. J Neurooncol 2011; 104 (1): 195-204.

32. Raverot G, Jacob M, Jouanneau E, Delemer B, Vighetto A, Pugeat M, et al. Secondary deterioration of visual field during cabergoline treatment for macroprolactinoma. Clin Endocrinol (Oxf) 2009; 70 (4): 588-92.

33. Cohen AR, Cooper PR, Kupersmith MJ, Flamm ES, Ransohoff J. Visual recovery after transsphenoidal removal of pituitary adenomas. Neurosurgery 1985; 17 (3): 44652.

34. Marcus M, Vitale S, Calvert PC, Miller NR. Visual parameters in patients with pituitary adenoma before and after transsphenoidal surgery. Aust N Z J Ophthalmol 1991; 19 (2): 111-8.
35. Sullivan LJ, O’Day J, McNeill P. Visual outcomes of pituitary adenoma surgery. St. Vincent's Hospital 1968-1987. J Clin Neuroophthalmol 1991; 11 (4): 262-7.

36. Powell M. Recovery of vision following transsphenoidal surgery for pituitary adenomas. Br J Neurosurg 1995; 9 (3): 367-73.

37. Peter M, De Tribolet N. Visual outcome after transsphenoidal surgery for pituitary adenomas. Br J Neurosurg 1995; 9 (2): 151-7.

38. Gnanalingham KK, Bhattacharjee S, Pennington R, Ng J, Mendoza N. The time course of visual field recovery following transsphenoidal surgery for pituitary adenomas: predictive factors for a good outcome. J Neurol Neurosurg Psychiatry 2005; 76: 415-9.

39. D'Ambrosio A, Syed O, Grobelny B, Freda P, Wardlaw S, Bruce J. Simultaneous above and below approach to giant pituitary adenomas: surgical strategies and longterm follow-up. Pituitary 2009; 12 (3): 217-25.

40. López-Arbolay O, Morales-Sabina O, González-González JL, Valdés-Lorenzo N. Transsphenoidal approach to prolactinomas. Neurocirugía (Astur) 2006; 17 (3): 226-31.

41. Valassi E, Klibanski A, Biller BM. Clinical Review: potential cardiac valve effects of dopamine agonists in hyperprolactinaemia. J Clin Endocrinol Metab 2010; 95 : 1025-33. 